\title{
Feasibility studies for a bionic propulsion system of a blimp based on Dielectric Elastomers
}

\author{
Silvain Michel*a, Alex Bormann ${ }^{\mathrm{b}}$, Christa Jordi $^{\mathrm{a}}$, Erich Fink ${ }^{\mathrm{c}}$ \\ ${ }^{a}$ Laboratory for Mechanical Systems Engineering, Swiss Federal Laboratories for Materials Testing \\ and Research (Empa), CH-8600 Duebendorf, Switzerland; \\ ${ }^{\mathrm{b}}$ AeroiX, Fichtenhof 5, D-14532 Kleinmachnow, Germany \\ ${ }^{c}$ Institute of Aeronautics and Astronautics, Technical University Berlin, Marchstrasse 12/14, D- \\ 10587 Berlin, Germany
}

\begin{abstract}
After having successfully integrated Dielectric Elastomers (DE) in a cross tail for flight control, a novel biologically inspired propulsion system based on DE is envisaged. The basic idea is to mimic a fish body motion by deforming a) the envelope of the rear lifting body and b) flapping an aft-tail. In both cases, planar DEs are used, either fully integrated in the envelope (for a) and/or arranged as an active hinge (for b). In a theoretical study the specifications of a steady-state horizontal indoor flight of $1 \mathrm{~m} / \mathrm{s}$ were defined. In an experimental work the concept of an active hull element, which consists of a balloon hull material and several layers of DE actuators was verified. The specific boundary conditions of a slightly pressurized elliptical membrane body were simulated in a biaxial test. It could be shown, that the necessary active strains to reach the specified body deformations were reached. In a second study an aero-elastic fin was designed. Based on fluid-dynamic similarity principles the size, shape and stiffness of the fin were determined and tested in preliminary flight test with a three meter long blimp. The main goal of $1 \mathrm{~m} / \mathrm{s}$ flight velocity could be shown.
\end{abstract}

Keywords: Dielectric Elastomers, Blimp, Lighter-than-Air vehicle, Biomimetics, Fish-like Propulsion

\section{INTRODUCTION}

Lighter-Than-Air vehicles are a promising class of transport systems for heavy cargo, stratospheric platforms, but also for surveillance or animal observations. In the last decades various concept studies have been performed for stratospheric platforms, which are interesting alternatives for land-based networks as well as satellites in telecommunication, reconnaissance and observation [1-4]. Classical propulsion systems composed of piston engines with gears and propellers show a limited efficiency due to thermo-mechanical losses in the engine, mechanical losses in the gears and fluid-dynamical restrictions of the propellers. In addition these systems are noisy and if driven by piston engines have a negative impact on the environment by pollution. These disadvantages can be grave for they prohibit some new applications such as stratospheric platforms (energy efficiency), animal observations or surveillance (noise). New systems should be energy efficient, noiseless and environmentally friendly. An idea in this context is to apply bionic principles.

\section{SYSTEMS DEFINITION AND SPECIFICATION}

The two main goals of the bionic EAP-driven airship project at the Swiss Federal Laboratories for Materials Testing and Research (Empa) are the following:

1) The Technology of Electro Active Polymers (EAP) and particularly the planar Dielectric Elastomers (DE) should be explored experimentally and shown practically in an indoor-flight model of a bionic airship. In his $\mathrm{PhD}$ study, Lochmatter has shown that shell-like DE-actuators can be successfully manufactured and integrated in a smart materials system, $[5,6]$. However, the system which was realized was limited to laboratory conditions. Therefore requirements for design space, weight and to certain extend also for environmental influences were not significant. In a next step, the technology should be developed for a more applied environment.

*Silvain.michel@empa.ch; phone +41-44-823 45 88; fax +41 4482340 11; www.empa.ch/eap

Electroactive Polymer Actuators and Devices (EAPAD) 2008, edited by Yoseph Bar-Cohen Proc. of SPIE Vol. 6927, 69270S, (2008) $\cdot 0277-786$ X/08/\$18 $\cdot$ doi: 10.1117/12.777588 
2) A Lighter-than-Air vehicle is an attractive practical example for a potential future application of EAPs and specially DEs. As a non-profit institute for material science and technology Empa can increase its reputation, if we can show successfully that EAP-technology can be integrated in a small airship. A small blimp is a challenge, because the size of the object is a critical parameter for such a project. While project development and operational costs are clearly increasing with size, the technical risk of feasibility is decreasing (with the exception of stratospheric platforms)! The development of a large airship, such as the Zeppelin NT (total length $80 \mathrm{~m}$ ?) is expensive and the operational costs are high. However the technical feasibility of such a large Blimp is less critical than for a smaller airship, because it is technically much more difficult to reach buoyancy with a small airship than with a large one. In a preliminary study we estimated that an airship of $6 \mathrm{~m}$ in length, $3 \mathrm{~m}$ in height and $2 \mathrm{~m}$ in width is feasible. Such an object is small enough, such that it can still be transported in a truck and be flown indoor. The fulfillment of requirements of design space and particularly weight and balance (weight distribution) are the major challenges and are critical for the entire project.

Based on these two major goals of the project, the technical specifications were defined in detail:

1. Form and motion, such that it is recognized as a fish-like object, e. g. at least a tail-fin stroke

2. The bionic propulsion system should be driven by EAPs

3. Buoyancy, radio controlled indoor free-flight up to $1000 \mathrm{~m}$ ASL

4. Steady-state cruising speed of at least $1 \mathrm{~m} / \mathrm{s}$

5. Radio controlled steering with conventional systems (Control surfaces with rudders)

6. Optical attractive design within the corporate design of Empa

7. Maintenance-free operation during at least 6 hours

8. Flight duration of at least 20 minutes

The following optional specification criteria were also defined:

9. Maximum cruising speed: The faster the better.

10. Acceleration and deceleration controlled by the tail-fin stroke.

11. Radio controlled steering by an asymmetric tail-fin-stroke.

12. Take-off and landing manoeuvres controlled by EAP-driven additional fins.

13. Out-door free flight in calm weather condition.

\section{THEORETICAL FEASIBILITY}

The method of mimicking biological forms and concepts in the development of technical products has a long tradition [7-9]. A pioneer in bionic aerospace design was Hertel, who already in 1963 was studying natural forms of various fishes for efficient fuselage designs and alternative propulsion systems for ships [8]. Later Bannasch analyzed in detail living penguins and their bodies, which have an extraordinary low drag coefficient [10]. His results were first used for an airship design by the Technical University of Berlin in 1998 in the airship called "Luftfisch". The shape of this $10 \mathrm{~m}$ long semi-rigid airship is basically a rotational symmetric shape of a penguin. We went one step further, when we suggested not only to adapt the form of a fish (in order to reduce the drag), but also its motion as an efficient propulsion system [11]. The main motivation for such a new concept for the propulsion of an airship is its much better efficiency. Hertel [8] predicted a power reduction of up to $60 \%$. It has been shown by Fish and Lauder that the efficiency of fish- 
like locomotion is significantly higher and less sensitive to variations of the coefficient of trust [12]. These interesting findings are not only valid in water, but when correctly transformed also in air.

If the shape and motion of swimming fish in water is to be transferred to an airship in air, the fluid-dynamic similarity theorem has to be considered.

\subsection{Similarity and dimensional analysis}

In the following analysis we assume a 6 meter long airship flying with a steady state velocity of $1 \mathrm{~m} / \mathrm{s}$ in a standard atmosphere $\left(23^{\circ} \mathrm{C}, 1023 \mathrm{hPa}\right)$. In order to be able to apply the locomotion mechanism of a fish in water to a deformable airship in air, the fluid-dynamic conditions as well as the geometrical shape of fish and blimp have to be similar. In the case of a fish locomotion in water and the motion of an airship in air, the two scenarios are fluid-dynamically similar, if the following non-dimensional parameters are equal [13]:
a. Reynolds number
b. Strouhal number

The Reynolds number can be calculated here with respect to the whole body length:

$$
\operatorname{Re}=\mathrm{v}_{\infty} L \frac{\rho}{\mu}
$$

Where $\mathrm{V}_{\infty}$ is $1 \mathrm{~m} / \mathrm{s}$ (the velocity of the airship), $\mathrm{L}$ is $6 \mathrm{~m}$ (it's length), $\rho$ is $1.0 \mathrm{~kg} / \mathrm{m}^{3}$ (density of air) and $\mu$ is $16.6^{*} 10^{-6}$ $\mathrm{kg} / \mathrm{ms}$ (dynamic viscosity of air). With these typical values for our case, we get a Reynolds number as follows:

$$
\mathrm{Re}=\mathrm{v}_{\infty} L \frac{\rho}{\mu}=1 \cdot 6 \cdot \frac{1.0}{16.6 \cdot 10^{-6}}=0.36 \cdot 10^{6}
$$

The Strouhal number is defined as:

$$
\mathrm{St}=\frac{D f}{\mathrm{v}_{\infty}}
$$

Where $\mathrm{D}$ is a typical dimension of the vortex street generated in the wake of the body, $\mathrm{f}$ is the stroke frequency and $\mathrm{v}_{\infty}$ is the cruising speed of the object. Because D is not an a priori known value, usually the Strouhal number is calculated with the peak-to-peak stroke amplitude of a reference point (the trailing edge of the tail fin) instead of $\mathrm{D}$. Other definitions have also been used, such as the depth of the tail fin [14], or even the full length of the fish [8]. Following Triantafyllou [15] we will us the Strouhal number based on the peak-to-peak amplitude, 2a:

$$
\mathrm{St}^{\prime}=\frac{2 a f}{\mathrm{v}_{\infty}}
$$




\subsection{The rainbow trout as a model for a 6 meter long airship}

From the various fishes we want to find one, which would be a meaningful model for our airship. The first condition of similarity is the Reynolds number of $0.36^{*} 10^{6}$. If we look for various species in water, we find for example the tuna and the rainbow trout, which are swimming with such a Reynolds number. This can be seen by the following calculation for the trout: The trout is $0.3 \mathrm{~m}$ long, and reaches a steady state swimming speed in water of $1.2 \mathrm{~m} / \mathrm{s}$. With L $=0.3 \mathrm{~m}, \mathrm{v}_{\infty}$ is $1.2 \mathrm{~m} / \mathrm{s}$, and $\rho$ is $1.0 * 10^{3} \mathrm{~kg} / \mathrm{m}^{3}$ and $\mu$ is $1.002 * 10^{-3} \mathrm{~kg} / \mathrm{ms}$ for water, the Reynolds number is:

$$
\operatorname{Re}=\mathrm{v}_{\infty} L \frac{\rho}{\mu}=1.2 \cdot 0.3 \frac{1.0 \cdot 10^{3}}{1.002 \cdot 10^{-3}}=0.36 \cdot 10^{6}
$$

Both are very efficient swimmers. Looking at the fish geometry as a design model, the rainbow trout seems to be a promising shape for the blimp because of its laminar formed body and the low sensitivity to cross winds. Additionally, the efficient propulsion is combined with a good maneuverability, which is also important for airships. On the other hand the tuna has an even more efficient lift-based propulsion mechanism compared to the trout, with a minimized tendency to side slipping and yawing of anterior body due to inertial recoil. However this fish is known to have a low efficiency at low speeds and bad maneuverability. Therefore we decided to use the rainbow trout as a design model for further studies in our airship design.

The shape of a rainbow trout has a width-to-body length ratio of approximately $14 \%$ and a height-to-body length ratio of approximately $22 \%$, see Fig. 1. (adapted from Hertel [8]). The maximum width and height is at $40 \%$ of the body length. This shape has a minimum of drag. Analytically, this shape can be approximated by a NACA0014-Profil in the x-y plane and NACA0022 in the x-z-plane. The body cross section can be assumed to be elliptical. In this way, we can take the trout as a model for the undeformed shape of the airship.

If we follow the paradigm of bionic, e.g. that nature has found not only optimized forms but also the corresponding optimized motion pattern; we can choose the motion pattern of the trout also for our airship in air. The motion pattern of a trout has been published for example by Webb [16]. We have analysed the deformed body of a trout in a steady-stateswimming mode, shown in Fig. 2.a.

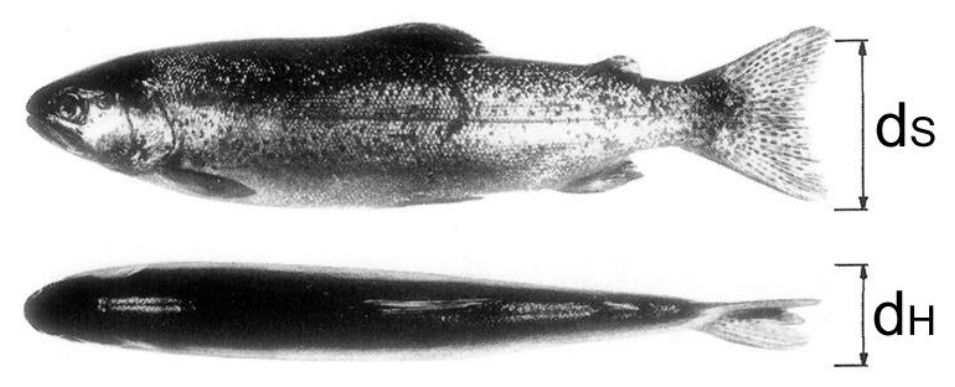

Fig. 1. Typical undeformed shape of a trout (adapted from Hertel [8]).

If we analyze the body motion of the steady state swimming we find the following typical values: The amplitude of the fin stroke is $20 \%$ of the length. The stroke frequency $\mathrm{f}$ is $4.5 \mathrm{~Hz}$ (see Hertel [8]) and the resulting swimming speed $\mathrm{v}_{\infty}$ is $1.2 \mathrm{~m} / \mathrm{s}$. With these values we find a Strouhal number of 0.45 . Analytically, the periodic deformation of the body can be represented by the superposition of the undeformed body contour with a time-dependent deformation field. A polynomial function representing the increasing lateral amplitude from the head to the tail can be defined, see for example Webb [16]. For simplicity in our case we used a quadratic function as a first order approximation. A detailed description of this time-dependent deformation field can be found in [13]. 
The adaptation of the fish-like locomotion mechanism in water to a deformable airship in air should be possible, if these geometrical and fluid-dynamical similarities are satisfied. The Reynolds number with respect to body length and the Strouhal number are the non-dimensional parameters of relevance to meet fluid-dynamical similarity. Therewith the caudal fin stroke frequency is exemplarily calculated: With $\mathrm{St}^{\prime}=0.45, \mathrm{a}=20 \%$ of $6 \mathrm{~m}=1.2 \mathrm{~m}$ and $\mathrm{v}_{\infty}=1 \mathrm{~m} / \mathrm{s}$. The stroke frequency is:

$$
f=\frac{S t^{\prime} \cdot v_{\infty}}{2 a}=\frac{0.45 \cdot 1}{2 \cdot 1.2}=0.1875 \cong 0.2 \mathrm{~Hz}
$$

Therefore we can conclude that a fully grown rainbow trout is a meaningful design model for the deformable airship.

\subsection{Preliminary Computational Fluid-dynamic Simulations}

The fish-like motion has been simulated with a NACA0012-profil with the help of the CFX software. The body shape and deformation defined above have been used to calculate the flow field around and in the wake of a fish-like moving profile. The Navies-Stokes equations have been solved in 2D for an incompressible, but viscous fluid. Figure 2.b shows the pressure distribution of the steady state solution after approximately 10 stroke cycles.

In this simulation, the vortex field in the wake of the body shows a very strong and clear reverse Kármán street with two vortices per cycle and a moderately strong leading edge vortex for angle of attack larger than $10^{\circ}$. This regime is limited by angle of attack values between $7^{\circ}$ and $50^{\circ}$ as well as Strouhal number values between 0.2 and 0.5 . The most efficient thrust production was found in this region by Anderson, too [17]. For a thrust coefficient of order one the maximum efficiency is found for Strouhal numbers between 0.3 and 0.4 with a maximum value of $87 \%$.

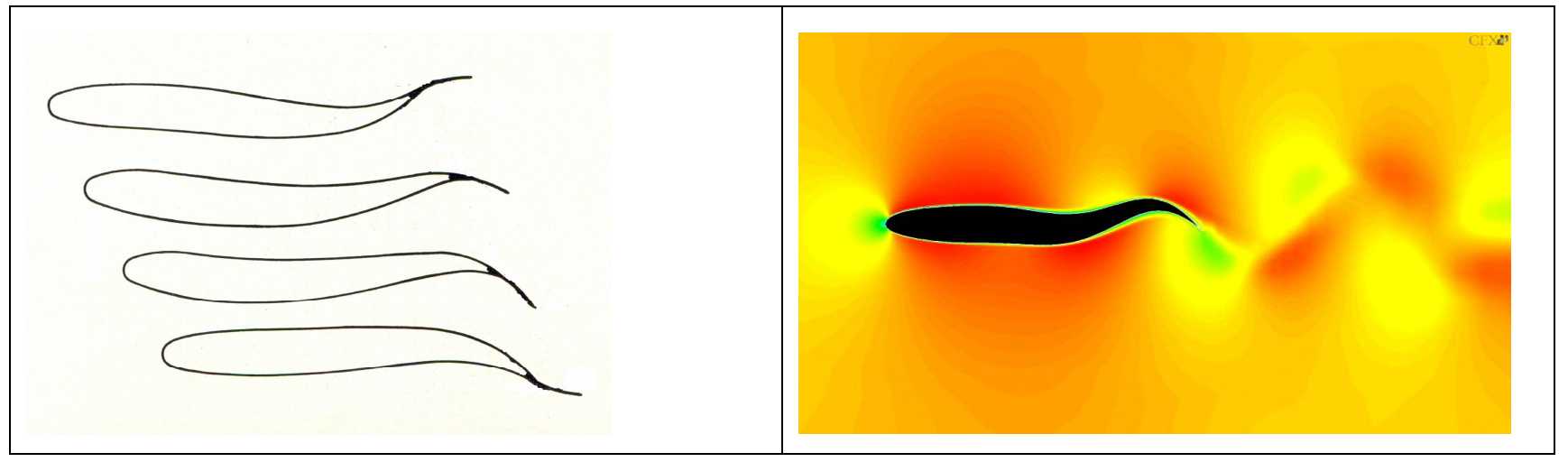

Fig. 2.a. Typical deformed shape of a trout in steady state swimming. The time step between individual pictures was $1 / 32 \mathrm{~s}$ (adapted from Hertel [8]).
Fig. 2.b. Pressure distribution around a 2D profile with undulatoric body motion.

\section{PRACTICAL FEASIBILITY - RESULTS OF DEVELOPMENT TESTS}

In the second part of the work parts of the bionic blimp with a fish-like propulsion system were developed. While the results of the development of an active hinge for driving a rudder in the control surfaces has been published $[18,19]$, the developments of the following subsystems have recently been completed and will be summarized here:

1. An active envelope.

2. A tail-fin for undulatoric propulsion in air.

In the following the results of these studies will be summarized. 


\subsection{The structural concept and verification of an active envelope}

In Figure 3 a schematic of the bionic blimp is shown. The body has different areas, which can contract (see arrows). A couple of two areas, one on each side, form a bending body segment. We intend to have at least two ob these bending body segments (e. g. four active areas) in our bionic blimp. By controlling these active areas individually, a simplified motion pattern of the trout body deformation discussed above can be realised. The required local strains in these active areas could be obtained through the application of Dielectric Elastomer (DE) membrane actuators on a conventional balloon hull material. The design of such a segment of the so called "active envelope" was studied theoretically and experimentally. The results of these studies will be discussed below.

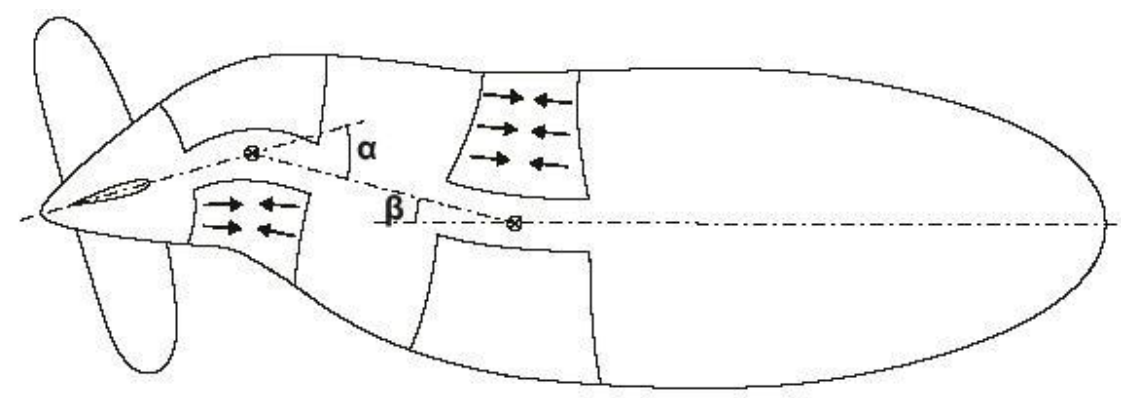

Fig. 3. Schematic of deformed shape of an airship, mimicking the motion of a trout.

Dielectric Elastomers are one material class of Electro active polymers (EAPs) and are promising material systems as actuators in active structures, where large deformations are required. They transform electrical energy directly to mechanical work and produce large strains (20\% and more). Especially Dielectric Elastomers were recently shown to have good overall performances. Pressures of $16.2 \mathrm{MPa}$, free area strains of $215 \%$ and specific elastic energy densities of $3.4 \mathrm{~J} / \mathrm{g}$ could be shown [20]. A DE actuator is basically a compliant capacitor, where a passive elastomer film is sandwiched between two compliant electrodes. When an electrical voltage is applied, the electrostatic forces arising from the charge displacement on the electrodes squeeze the elastomer film. This electrode pressure mechanically loads the polymer film. Because such elastomers are essentially incompressible the area of the capacitor is enlarged. As soon as the voltage is switched off and the electrodes are short-circuited the capacitor contracts back to its original shape.

The so call "active envelope" is the combination of a Dielectric Elastomer (DE) membrane actuator mounted on a balloon hull. The balloon hull is a lightweight and helium-tight membrane, which is characterized by a high tensile strength and a high compressive compliance. The DE-actuator consisting of at least one prestretched dielectric film layer coated on both sides with compliant electrodes. We use VHB- $4910^{\circledR}$ from $3 \mathrm{M}^{\odot}$ as dielectric material and carbon black powder (Ketjen Black 600) as compliant electrodes. The combination of these two subsystems results in an active envelope. The principles of this active structural element is illustrated schematically in a force-versus-deformation diagram, see Fig. 4.a. During the manufacturing process the over-stretched DE-actuator is attached directly onto the blimp envelope (point 1). If the actuator exerts a resulting tension force larger than that induced by the internal pressure in the envelope, the membrane below the DE-actuator will wrinkle (from point 1 to 2 on the line denoted as "passive DE”). By applying an electrical voltage to the DE-actuator, the DE actuator is enlarged and the envelope segment regains its fully elongated state (point 3 on the line denoted as "active DE"). The slope of both lines represents the stiffness of the DE actuator. If the internal pressure is too high, then the envelope remains stretched and the stiffness of the system is the stiffness of the envelope (steep line beyond point 1). Therefore it is important to adjust the internal pressure such that the full deformation range (difference between point 2 and 3 ) is possible. 


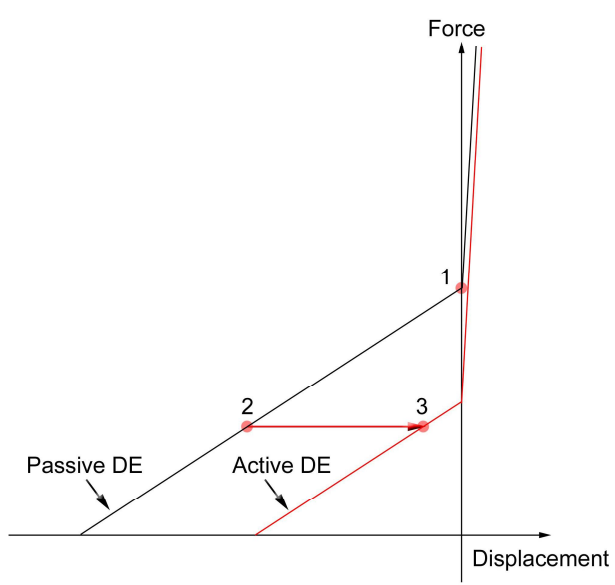

Fig. 4.a. Force versus displacement behaviour of an active segment of the envelope.

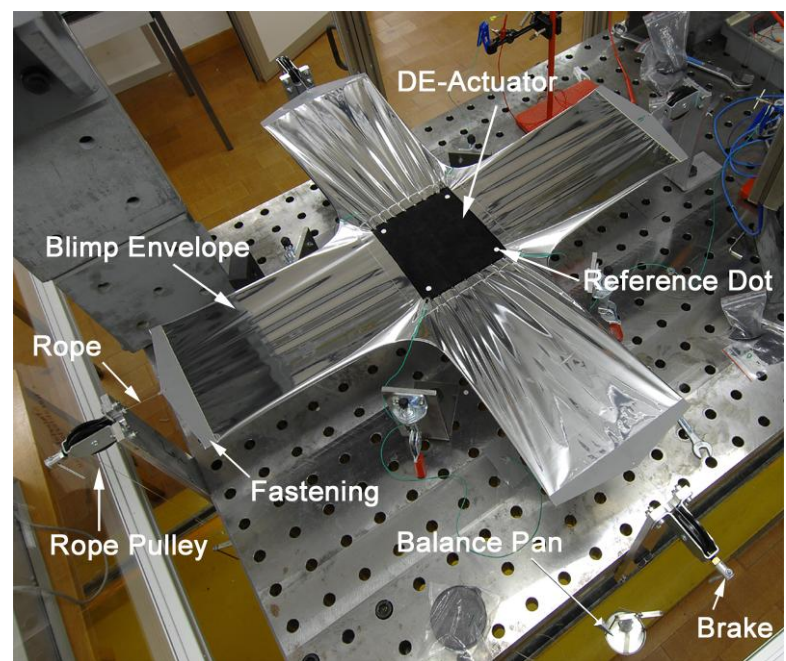

Fig. 4.b. Biaxial tension test set-up for the active segment of the envelope.

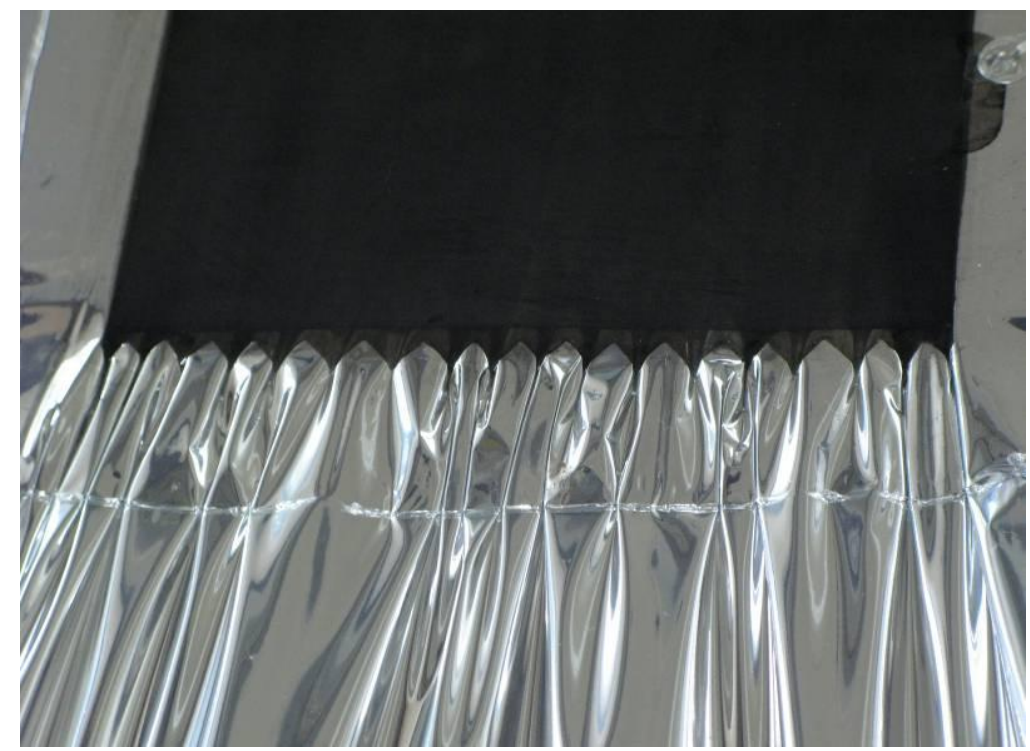

Fig. 4.c. Detail of Figure 7.b: The wrinkling of the balloon envelope under the EAP-actuator is clearly visible.

In a theoretical study the membrane stresses and strains of such an "active envelope" for a blimp body slightly pressurized and deforming like a trout have been analyzed. In order to experimentally simulate the stress state of the blimp envelope, a functional model of the active envelope was defined and experimentally characterized. With this cruciform planar functional model mounted on an in-house made testing device the deformations on activation under representative membrane stresses could be measured, see Fig. 4.b. The feasibility of the proposed concept could be investigated. The line loads on each arm of the cross were determined to represent the stress distribution in the slightly pressurized blimp (around $300 \mathrm{~Pa}$ ). These line loads were calculated for a $6 \mathrm{~m} \mathrm{x} 3 \mathrm{~m}$ x $1 \mathrm{~m}$ ellipsoidal body. The line loads vary with orientation and location from $160 \mathrm{~N} / \mathrm{m}$ in the longitudinal direction at the thickest section to zero at the nose and the tail. The circumferential line load varies from $80 \mathrm{~N} / \mathrm{m}$ to zero respectively. The ratio between longitudinal and circumferential line loads is between 1 and 2. Furthermore, the envelope strain and strain rate requirements for steady (and accelerated) swimming were also defined. The required local deformations of the envelope were derived from the 
analysis of the deformed fish body. The maximum strain from one reversal point of the other was $14 \%$ in longitudinal direction for the steady state swimming mode.

The envelope material Nonex ${ }^{\circledR}$ (detailed description, see below) satisfies the basic mechanical and aerostatic requirements. Thanks to its small thickness $(25 \mu \mathrm{m})$, it can be wrinkled easily. The metal coating guarantees the required Helium tightness. The envelope can be cut in segments that are successively welded together in order to achieve a Helium-tight body with high compliance under compression forces.

The largest longitudinal activation strain was achieved by the active envelope with a $\left(\left(\lambda_{\mathrm{x}}=6, \lambda_{\mathrm{y}}=3\right.\right.$, $)$-over-stretched $\mathrm{DE}$ actuator. It reached $28.62 \%$ at an activation voltage of $3.5 \mathrm{kV}$. This was reached under a biaxial line load ratio of 2.36 . This quasi-static strain satisfies the lateral strain requirement of $14 \%$ for steady swimming. Furthermore, the reduction of the transversal load not only allows to increase the longitudinal activation strain, but also to decrease the line load ratio from 2.37 to 2 . Thus, the requirement for the blimp line load ratio could be fulfilled. In order to verify the assumption that the forces are linearly increasing with the number of layers tests with two and three layers were also made. The forces increased as expected and no significant impact on the activation strains was found. The membrane line load requirement of $160 \mathrm{~N} / \mathrm{m}$ at $\Delta \mathrm{p}=300 \mathrm{~Pa}$ and $80 \mathrm{~N} / \mathrm{m}$ can be fulfilled through the stacking of eight DE-actuators.

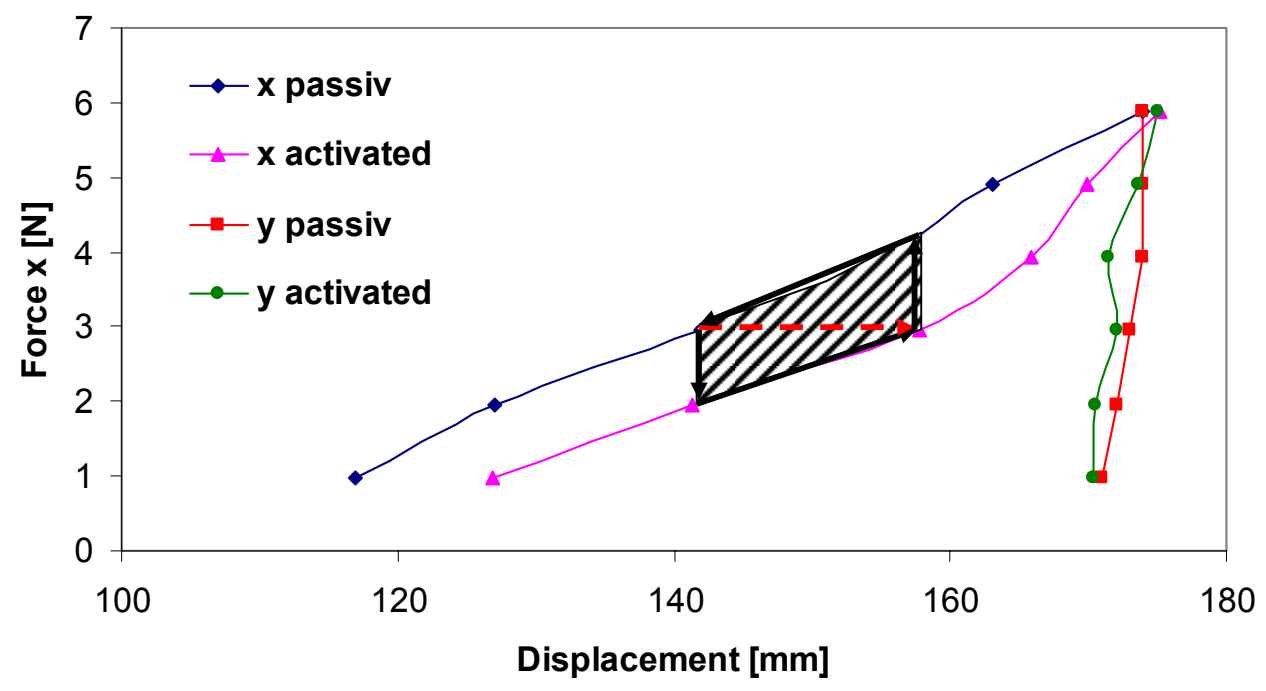

Fig. 5. Static force versus displacement diagram of a segment of the active envelope in the biaxial test.

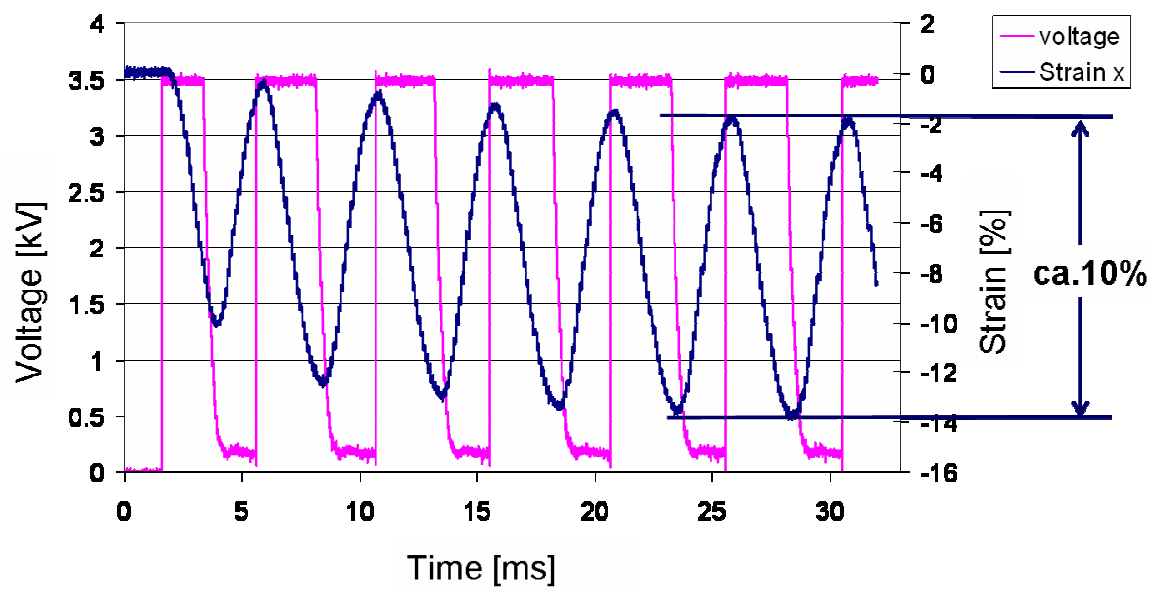

Fig. 6. Activation voltages and strain response of a segment of the active envelope in the biaxial test 
In the first tests large scattering of results of samples with the same configuration was found and therefore reproducibility was not fully satisfactory. By analysing the various factors of the manufacturing process, influencing the characteristics of these samples, a main cause of this scatter was found in the wrinkling behaviour. By choosing a zickzack boundary of the electrodes, the wrinkling pattern could be predefined, see Fig. 7c. With this important detail, the reproducibility could be improved significantly.

In order to get a first estimation of the efficiency current measurements were carried out during an activation cycle from 0 to $3.5 \mathrm{kV}$. The current was measured with a Shunt of $50 \mathrm{M} \Omega$. The electrical energy stored in the DE-actuator was calculated by integrating the current peak multiplied by the voltage $(3.5 \mathrm{kV})$. The mechanical work of a working cycle was estimated by the force times the displacement both in the $\mathrm{x}$-direction. The efficiency as defined by Lochmatter in [21], was calculated. Dependent on the samples the electro-mechanical efficiency was between $4.1 \%$ and $14.8 \%$ (average $9.65 \%)$.

The dynamic response of the DE-actuators was first determined by measuring the strain rates due to an instantaneous increase of the voltage from 0 to $3 \mathrm{kV}$. A typical strain rate of $1.8 \% / \mathrm{s}$ was found at moderate loads, which is equivalent to a mechanical power of $257 \mathrm{~mW} / \mathrm{m}^{2}$. In other specimens strain rates of up to $3.5 \% / \mathrm{s}$ were measured, however these specimens were significantly less loaded. Therefore a mechanical power of $250 \mathrm{~mW} / \mathrm{m}^{2}$ can be seen as a typical value for further preliminary design calculations.

The dynamic response was also characterized in repeated activation cycles. From the fluid-dynamically similarity a frequency of $0.2 \mathrm{~Hz}$ was chosen. A voltage of $3.5 \mathrm{kV}$ was turn on and off one in 5 seconds, sees Figure 6 . After a few cycles at a slightly higher mean level (maximum range 12\%), the strain oscillated with a range of approx. 10\%. Although we do not yet achieve the specified value of $14 \%$ with $3.5 \mathrm{kV}$, we expect to reach this goal by activating with higher voltages.

These experimental results allow concluding that the proposed concept for an active envelope is feasible at least for the steady swimming mode.

\subsection{The tail fin design and development}

There is a limited number of projects on oscillating foils or fins and to the knowledge of the authors they have been done all in water, see for example Anderson, et al. [17] and [22]. We have studied the design of an aero-elastic tail fin as a second element of the undulatoric propulsion. Again with the theory of similarity it can be shown, that an elastic tail fin of such a 6 meter long airship is similar to a tail of a trout in water, if the dimensions of the fin and the deformation is geometrically equivalent. In Figure 7.a rainbow trout is shown with typical dimensions of her tail fin: The shape is a triangle with a sweep angle of $25.2^{\circ}$ and a length of approximately $25 \%$ of the total length.

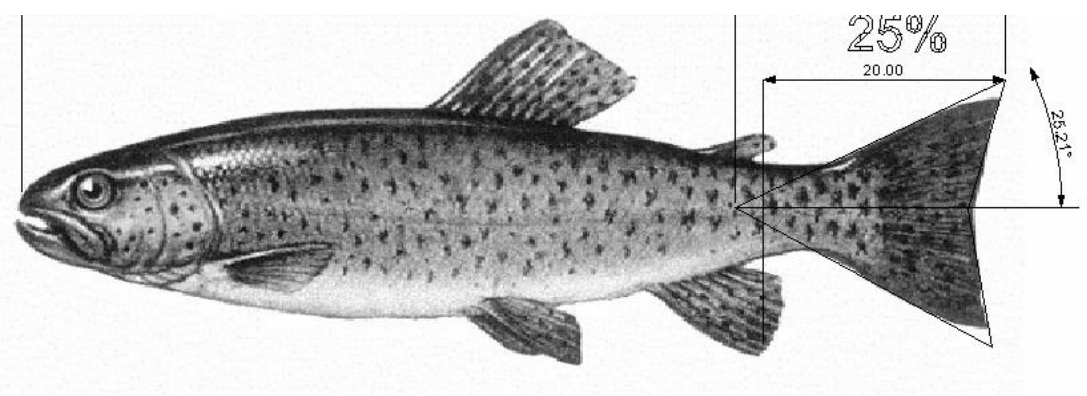

Fig. 7. Typical shape and size of the rainbow trout tail fin.

The theoretical aspects of the transformation of a flexible structure generating undulatoric propulsion in water to air were studied. The main result was that the aero-elastic problem can be reduced to a structural one. If similarity is fulfilled not only with respect to the size and shape of the fin, but also with respect to its motion (same deformation), the flow field in the wake of the body will be the same. The same vortex-structure will be generated and the same propulsion effects can be expected. 
The practical aspects of this problem were studied in experiments with tree different types of triangular shaped fins (see Figure 8.a), which were oscillated in a wind flow. With these preliminary tests it could be showed, that the deformation of the fins can be monitored by a high speed camera and the deformation can be measured accurately. By varying the stiffness of the fin structure the maximum deformation of the fin in a wind flow could be defined, see Figure 8.b.
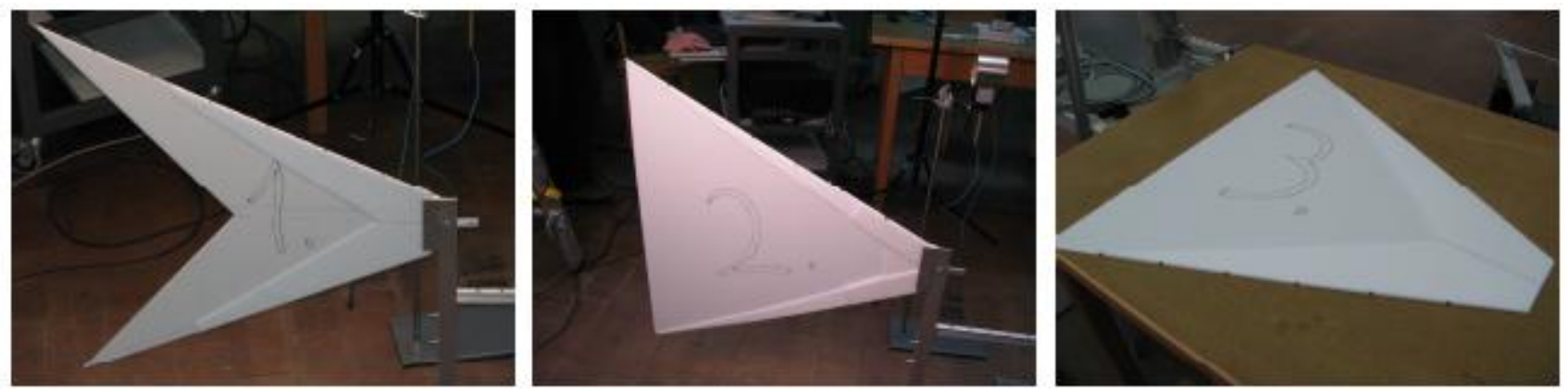

Fig. 8.a. Three types of triangular shaped tail-fins.
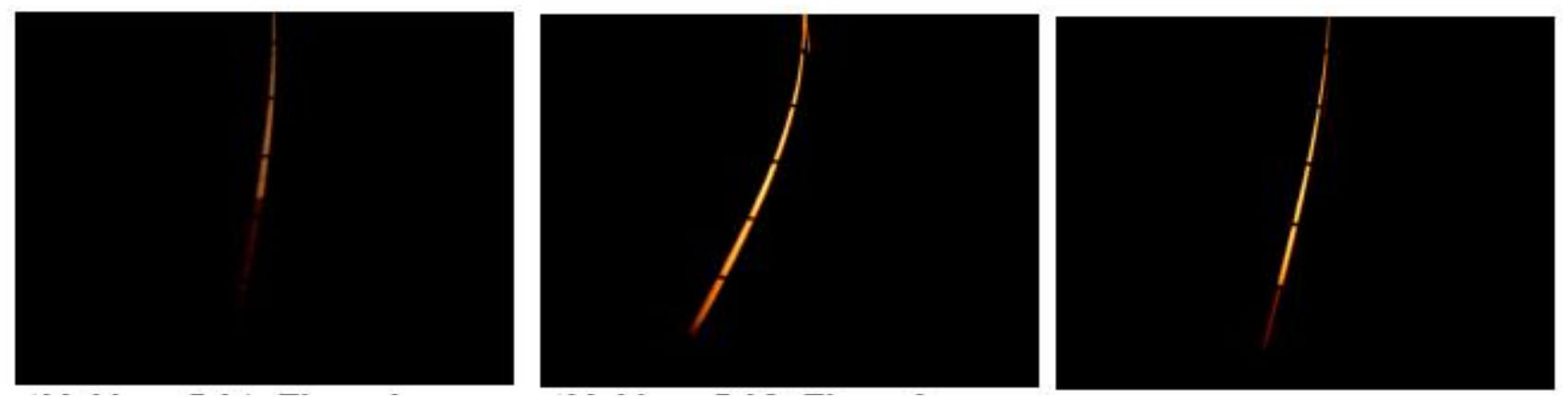

Fig. 8.b. The maximum deformation of the fins shown in Fig. 8.a, view from top.

\subsection{Experimental verification with functional models}

Two functional models of a blimp, one with a conventional and one with a bionic propulsion system were realized, see Figure 9.a and 9.b. respectively. In both cases, the blimp envelope was identical in size and shape to allow for a direct comparison. The payload gondola is a three quarter closed box made of Depron ${ }^{\circledR}$ with one main spar in the centre. All the electrical equipment was placed within the gondola, except the power supply accumulator, which was fixed as an additional external payload, positioned as a balance weight. For controlling the EAP blimp a commercial radio control (RC) unit which is normally used for model airplanes or model helicopters was chosen. It is equipped with control sticks for altitude control, direction control, and also for speed control. 

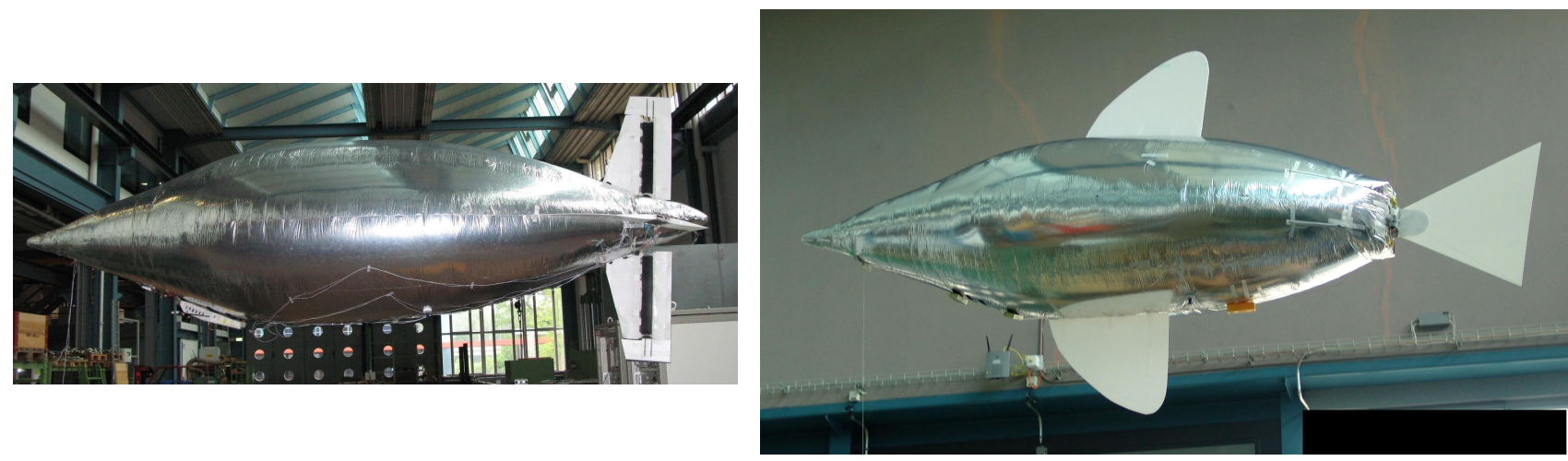

Fig. 9.a. Conventional model blimp propelled by electric DC Figure 9.b. Bionic model blimp with undulating tail-fin as the motors and propellers, with improved cross-tail driven by propulsion and steering system

EAP active hinges

The first functional model (conventional, Version "C20") was an improved version of the airship shown at the EAP-inaction session at the SPIE-EAPAD conference in 2007 (Version "C10"). This type of a conventionally propelled blimp had a cross tail with active hinges. With this model, the successful integration of shell-like EAPs in a Lither-than-Air vehicle could be shown [18]. However, two problems were not yet solved properly: First, the electrical reliability was a problem in some flight demonstrations and second, the model had not yet a satisfactory agility and therefore the maneuverability was limited. In a redesign study both problems were evaluated. With the improved version both problems could be solved to our satisfaction. The electrical problem was due to the electrical wiring along the lifting body, which led to unforeseen electrical interactions between the different actuator circuits. By positioning the different wiring apart, this problem was quickly and fully solved. The problem of the unsatisfactory agility needed more effort and could only be solved by a complete redesign of the cross tail. The main modifications were: First, the control surfaces were significantly reduced. Secondly, the rudders were modified with balance areas, which reduced the driving moment. Third, for the elevator asymmetrically multilayer active hinges (3 layers on top and 1 layer on the lower side) were installed. The electromechanical aero-dynamical characteristics of the original, as well as the redesigned cross tail were evaluated in the wind tunnel at ETH in Zürich. With the redesigned model (Version "C20"), a much better agility could be reached. This model will be shown at the EAP-in-action session at the SPIE-EAPAD conference in 2008.

Table 1. Weight and balance of improved conventional model blimp with EAP driven cross tail (Version "C20”).

\begin{tabular}{|c|c|c|}
\hline Component & $\underline{\text { Structural weight }}$ & Position from tip \\
\hline Envelope & $192 \mathrm{~g}$ & $174 \mathrm{~mm}$ \\
\hline Gondola, with engines, electronics & $254 \mathrm{~g}$ & $100 \mathrm{~mm}$ \\
\hline Cross tail with EAP actuators & $207 \mathrm{~g}$ & $300 \mathrm{~mm}$ \\
\hline Wires & $43 \mathrm{~g}$ & $220 \mathrm{~mm}$ \\
\hline Power supply (LiPo accumulator) & $88 \mathrm{~g}$ & $55 \mathrm{~mm}$ \\
\hline Front balance weight & $31 \mathrm{~g}$ & $30 \mathrm{~mm}$ \\
\hline Payload (weight) & $81 \mathrm{~g}$ & $200 \mathrm{~mm}$ \\
\hline Total weight $(=$ aerostatic lift) & $910 \mathrm{~g}$ & $171 \mathrm{~mm}(\mathrm{CG})$ \\
\hline
\end{tabular}

The second functional model (bionic, or "B"-Versions) was developed in order to verifying the practical feasibility of the undulatoric propulsion concept in air. While the theoretical feasibility has been discussed in detail in $[11,13]$ the practical feasibility was still an open task. There is only a very limited number of airships that are propelled by a fish-like propulsion system: The first one is the "Air_ray" from FESTO, [23]. This free-flying object was a semi-rigid hybrid airship, with a lifting body filled with Helium in the centre section and flapping wings on each side. This airship was looking like and mimicking successfully the motion of a stingray. It was first shown at the "Hannovermesse" in 
Hannover in 2007, [24]. The second one is a model blimp called "Wanda" shown by Martin Müller at the Wall-Halla indoor flying meeting 2008, [25]. However, none of these objects have been investigated scientifically.

Our bionic airship allowed to exploring scientifically the bionic propulsion system in flight tests. This model was equipped with a flexible fish-like tail-fin instead of the cross-tail. A small tuna-shaped tail fine adopted from the fish "Scomber Japonicus ", and two sizes of the trout-like tail fin were tested. The small one was identical in size and shape as the type 2 from the tail-fin study. The large one was scaled correctly in respect to the relative size of the tail-fin shown in Fig. 7. These tail-fins were mounted with a hinge at the end of the body, see Fig. 10. With a conventional servo motor this tail-fin was driven such that an undulatoric flapping motion of the root of the fin was realized. Various amplitudes $\left( \pm 12.5^{\circ}, \pm 20^{\circ}, \pm 30^{\circ}\right)$ and frequencies $(0.5-2 \mathrm{~Hz})$ could be applied. In total eight different fins, with different shapes, sizes and stiffness were tested in flight, see table 2 . The flight motion was qualitatively judged with respect to stability and the cruising speed was measured.

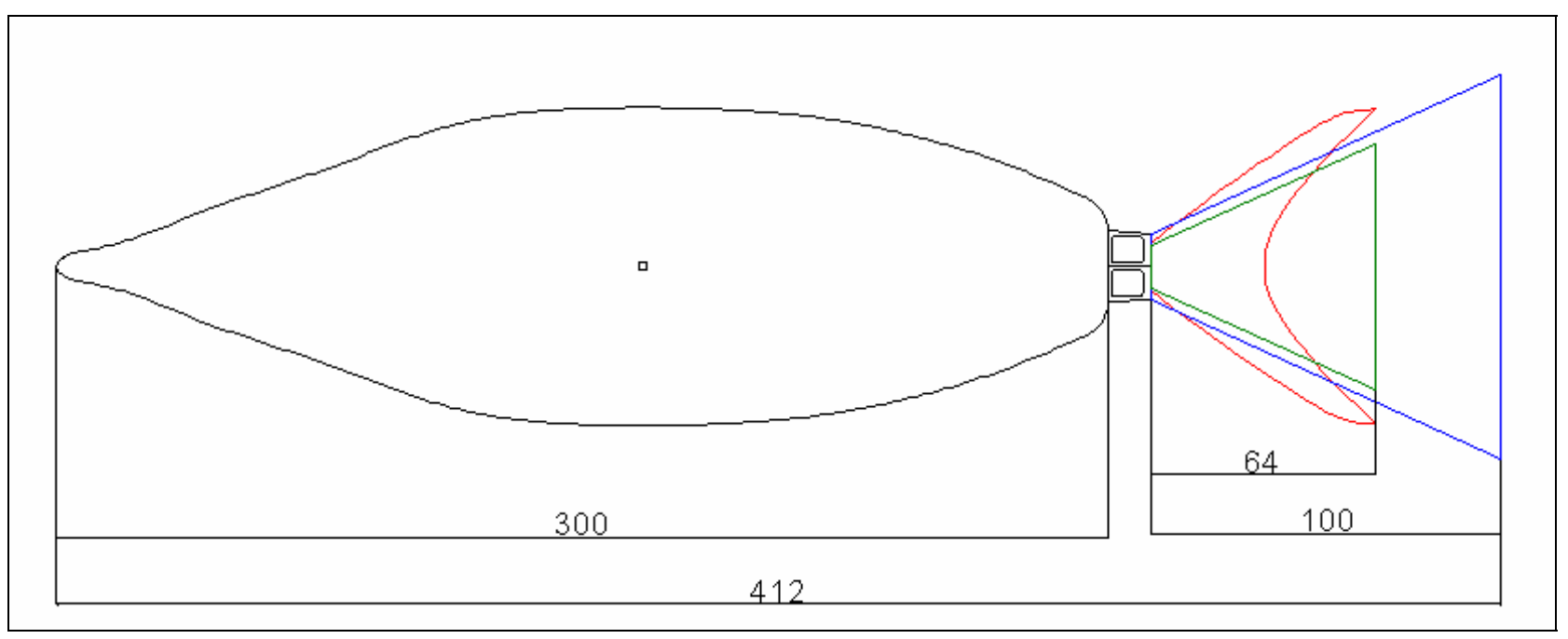

Fig. 10. Shape and size of the tail-fins tested in flight tests.

Table 2: Summary of flight tests with various tail-fins: The last digit is a ranking number indicating the stiffness level from low to high.

\begin{tabular}{|ccc|}
\hline Type of tail fin & $\begin{array}{c}\text { Stiffness level } \\
\text { (Version) }\end{array}$ & Maximum cruising speed \\
tuna $(\mathbf{l = 0 , 6 4 m )}$ & B10 & $0,77 \mathrm{~m} / \mathrm{s}$ \\
& B11 & $0,99 \mathrm{~m} / \mathrm{s}$ \\
rainbow trout $(\mathbf{l}=\mathbf{0 , 6 4 m )}$ & B20 & $0.85 \mathrm{~m} / \mathrm{s}$ \\
& B21 & $1,15 \mathrm{~m} / \mathrm{s}$ \\
& B22 & $1,29 \mathrm{~m} / \mathrm{s}$ \\
& B23 & $1,43 \mathrm{~m} / \mathrm{s}$ \\
rainbow trout $(\mathbf{l = 1 , 0 m )}$ & B24 & $1,41 \mathrm{~m} / \mathrm{s}$ \\
\hline
\end{tabular}

The effect of shape, size and stiffness on the speed of the blimp at various Strouhal-Numbers (given by the frequency and the amplitude) could be quantified. All of these design parameters have a significant effect on the cruising speed. The speed is increasing with increasing frequencies (and increasing amplitude) as well as with increasing stiffness of the tail-fin, sees Fig. 11. With the triangle shaped, $1 \mathrm{~m}$ long tail fin a maximum speed of $1.55 \mathrm{~m} / \mathrm{s}$ could be reached at a flapping frequency of $1.4 \mathrm{~Hz}$ and a peak-to-peal-amplitude of $0.11 \mathrm{~m}$ (Strouhal number $=0.2$ ). This speed satisfies the minimum speed requirement given in specification, see chapter 2 . 


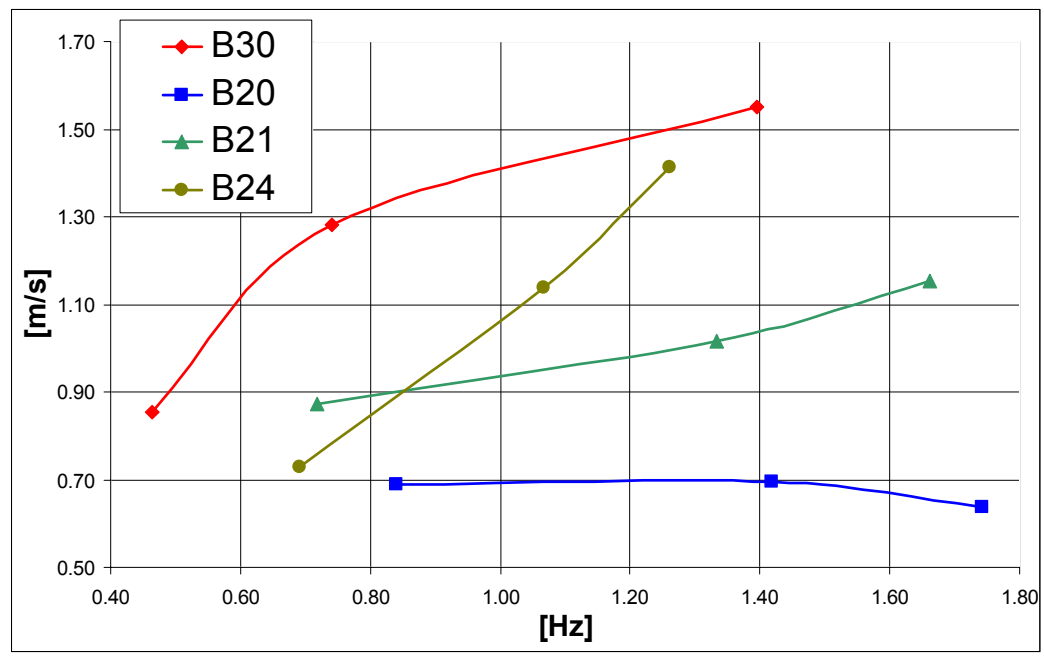

Fig. 11. Shape and size of the tail-fins tested in flight tests.

As Wiguna has reported for fish-swimming in [26], we can also conclude for our bionic blimp, that the total area and the aspect ratio have a significant influence on the speed. McHenry [27] concluded from fish observations, that fish do control their speed by varying their body motion pattern. In the design of the bionic blimp the resulting motion pattern is crucial for an optimal speed. Therefore the stiffness of the fin plays an important role in the design of a bionic blimp.

\section{OPEN QUESTIONS AND FUTURE WORK}

The major task in future work will be the structural technology of the active body. Detailed design studies on structural configurations and the definition of the distribution of active hull segments to generate the required bending deformation have already started. One problem in this field is the missing Finite Element tool, which would allows us to simulate physically correctly the stresses and strains/deformations in EAP-activated membrane or shell structures. Wissler has solved the material modeling for the material VHB-4910 from 3M and has successfully integrated this material model in a FE-environment, [28]. These FE-simulations are limited to cases, where volumetric FE-elements can be used. For a structure like the active airship body however, it is obligatory to use membrane or shell elements due to computational hardware limitations. This is so far not yet possible. A shell-element with EAP-characteristics is now under development. Structural analysis and experiments have shown that a semi-rigid internal structure is needed. In a first series of experiments with such semi-rigid bending body segments, the required bending angle could be reached. However the manufacturing process for these active segments is still unsatisfactory. The primarily hand-made active hull segments show still a rather poor reliability and the reproducibility has to be improved significantly by using industrial fabrication processes.

Computational Fluid-Dynamics (CFD) have started. We can expect realistic results from simulations of the fish-like motion. Nowadays, with the help of very powerful computer resources and novel optimization methods in fluiddynamics the 3D vortex structure behind the fish body can be simulated, see for example [29]. However such simulations are limited to simplified body geometries with predefined boundary conditions. In other words the structure of the fishbody is assumed to be elliptical and ideally rigid. If we have a more complicated 3D shape of the body made of soft tissue or very flexible structures, such as fins the complex structural-fluid-coupling is still an open issue. Structure-fluidinteractions can nowadays be simulated for linear-elastic structures, with only small deformations in a stationary stream. The instability effects of structural vibrations (normally flexural-torsional modes of an airfoil) exposed to the stationary flow of the fluid can only be simulated by linearizing the deformations and therefore the coupling forces. The instationary flow fields are embedded in a predominantly stationary flow. In our case, we have predominantly instationary flow fields around a flexible structure, drifting slowly away from it. In the case of the tail fin having FinRay characteristics such coupling effects are even more complex, and can by no means be ignored. In this case we have a strong influence of the structure on the flow field and vice-versa. 
Due to the fact, that we have not yet the methods available to fully simulate the structural mechanics and the fluiddynamics the empirical approach is very important in this project. We will continue to explore the structural behavior of active membranes in experiments, as well as the fluid-dynamic performances of the fins and the whole airship in wind tunnel tests or flight trials. Optimization of the whole system "bionic airship" will be the subject of longtime research, also in our group.

\section{CONCLUSIONS}

The proposed radical new concept of a bionic blimp is a multidisciplinary challenge. Many aspects, such as aerodynamics, aerostatics and active structures have to be fulfilled in order to be a functional solution. For many of these design criteria the methods have not yet be developed. However, if there is a combination of active hull segments with a geometrical configuration fulfilling the boundary conditions much more energy efficient, noiseless and environmental friendly transport systems would be possible.

\section{ACKNOWLEDGMENTS}

The fluid-dynamic studies done by Daniel Krämer, the CFD-simulations done by Simon Bucheli and Simon Frey, the study on aero-elastic fins done by Stefan Schori, the wind tunnel tests with the $1 / 4^{\text {th }}$ of a cross tail done by Martin Zobel and Stefano Airaghi, the support from Lukas Prochazka and Martin Wähmer in the wind tunnel tests with the oscillating tail fins at the Institute of Fluid-dynamics at ETH Zurich is greatly acknowledged. We thank Leif Kniese from Evologics $\mathrm{GmbH}$ for the design, manufacturing and delivery of a FinRay-Fin and the board of directors from Empa for the financial founding of this work. 


\section{REFERENCES}

[1] Warwick, G., "Return of the Airship," Flight International 15. - 21. August, (2006).

[2] Apel, U., "Stratospheric Platforms a definition study for a ESA platform," ESA, (2005).

[3] http://www.sanswire.com [cited Sep 10th 2007 ]

[4] http://www.gefa-flug.de [cited Sept 10th 2007]

[5] Lochmatter, P., ETH Zurich, Diss. ETH No. 17221, 2007.

[6] Lochmatter, P., Kovacs, G., and Ermanni, P., "Design and characterization of shell-like actuators based on soft dielectric electroactive polymers," Smart Materials and Structures 16 (2007), (2007), 8.

[7] Nachtigall, W., Bionik - Grundlagen und Beispiele für Ingenieure und Naturwissenschaftler. (Springer, Berlin, Heidelberg, New York, 1998).

[8] Hertel , H., Struktur - Form - Bewegung. (Krauskopf-Verlag, Mainz, 1963).

[9] Bar-Cohen, Y. and Breazeal, C., Biologically Inspired Intelligent Robots. (SPIE Press, Bellingham, Washington USA, 2003).

[10] Bannasch, R., 1. Int. Airship Conference, Stuttgart, 1993 (unpublished).

[11] Michel, S., Kovacs, G., and Lochmatter, P., CH Patent No. WO 2006/108311 A1 (19. 10. 2006 2006).

[12] Fish, F. E. and Lauder, G. V., "Passive and Active Flow Control by Swimming Fishes and Mammals," Annu. Rev. Fluid Mech. 38, (2006), 193-224.

[13] Michel, S., Bernasconi, M., Bormann, A., Zobel, M., and Fink, E., AIAA LTA, Belfast, 2007 (unpublished).

[14] Sfakiotakis, M., Lane, D. M., and Davies, J. B. C., "Review of fish swimming modes for aquatic locomotion," IEEE J of Oceanic Engineering 24 (2), (1999), 237-711.

[15] Triantafyllou, M. S., Triantafyllou, G. S., and Yue, D. K. P., "Hydrodynamics of Fishlike Swimming," Annu. Rev. Fluid Mech. 32, (2000), 33-53.

[16] Webb, P. W., "Is the High Cost of body/caudal fin undulatory swimming due to increased friction drag or inertial recoil " $J$. of Exp. Biology 162, (1992), 157-166.

[17] Anderson, J. M., Streitlien, K., Barrett, D. S., and Triantafyllou, M. S., "Oscillating Foils of High Propulsive Efficiency," J. Fluid Mech. 360, (1998), 41 - 72.

[18] Michel, S., Dürager, C., Zobel, M., and Fink, E., SPIE EAPAD, San Diego, USA, 2007 (unpublished).

[19] Lochmatter, P. and Kovacs, G., "Design and Characterization of an active hinge segment based on soft dielectric EAPs," Sensors and Actuators A: Physical 141 (2008), (2007), 11.

[20] Bar-Cohen, Y., Electroactive polymer (EAP) actuators as artificial muscles : reality, potential, and challenges. (SPIE Press, Bellingham, Washington, 2004), 2nd ed.

[21] Lochmatter, P., Kovacs, G., and Michel, S., "Characterization of dielectric elastomer actuators based on a hyperelastic film model," Sensors and Actuators A: Physical 135 (2007), (2007), 748 - 757.

[22] Bandyopadhyay, P. R., "Trends in Biorobotic Autonomous Undersea Vehicles," IEEE JOURNAL OF OCEANIC ENGINEERING 30 (1), (2005), 109 - 139.

[23] http://www.festo.com/INetDomino/coorp_sites/en/c79c5d07d5805095c12572b9006f04f5.htm [cited Feb 19th 2008]

[24] http://www.hannovermesse.de/homepage e [cited Feb 19th 2008]

[25] http://www.wall-halla.de/index.html [cited Feb 19th 2008]

[26] Wiguna, T., Park, H. C., Heo, S., and Goo, N. S., SPIE EAPAD, San Diego, 2007 (unpublished).

[27] McHenry, M. J., Pell, C. A., and Long, J. H., "Mechanical Control of Swimming Speed -Stiffness and Axial Wave-Form in Undulating Fish Models," Journal of Experimental Biology 198 (11), (1995), 12.

[28] Wissler, M., PhD, ETH Zurich, Diss. ETH No. 17142, 2007.

[29] Kern, S. and Koumoutsakos, P., "Simulations of Optimized Anguilliform Swimming," Journal of Experimental Biology 209, (2006), $4841-4857$. 